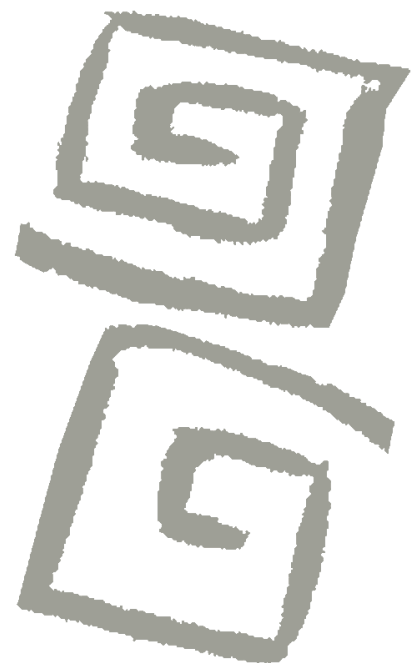

\title{
Aportes a una antropología feminista de la salud: el estudio del ciclo menstrual
}

\author{
Contributions to a feminist anthropology of health: \\ the study of the menstrual cycle
}

Maribel Blázquez Rodríguez', Eva Bolaños Gallardo²

'Doctora en Antropología. Profesora de Antropología Social, Departamento de Antropología Social, Universidad Complutense de Madrid, España. $\square$ (iD

${ }^{2}$ Psicóloga. Investigadora independiente. España.

$\triangle$ (iD)
RESUMEN La antropología médica o de la salud centrada en el estudio de las mujeres sigue constituyendo una de las áreas principales del quehacer antropológico en el Estado español. A partir de los aportes de dos referentes de la antropología feminista de la salud como Marcia Inhorn y Mari Luz Esteban, se revisan los hallazgos de una investigación cualitativa sobre del ciclo menstrual, en la que se realizaron 20 entrevistas a mujeres de 16 a 44 años, entre 2013 y 2014, en el municipio de Madrid. El análisis muestra cómo la menstruación es un claro ejemplo de la esencialización reproductiva de las mujeres, del reduccionismo biológico, de la medicalización de los cuerpos de las mujeres y, sobre todo, de su uniformización. La visibilización y cuestionamiento de estos supuestos, a través de las voces de las mujeres entrevistadas, pone de manifiesto la relevancia de este campo dentro de la antropología médica.

PALABRAS CLAVES Menstruación; Feminismo; Antropología Médica; Salud de la Mujer; España.

ABSTRACT Medical or Health Anthropology focused on the study of women continues to be a main area of anthropological study in Spain. The contributions of two referential figures in feminist health anthropology, Marcia Inhorn and Mari Luz Esteban, are applied to review the findings of a qualitative research study on the menstrual cycle carried out through 20 interviews with women between the ages of 16 and 44 years, between 2013 and 2014, in the municipality of Madrid. The analysis shows that menstruation is a clear example of the reproductive essentialization of women, of biological reductionism, of the medicalization of women's bodies and, above all, of the standardization of bodies. The visibilization and questioning of these assumptions through the voices of the women interviewed highlight the importance of this field within medical anthropology.

KEY WORDS Menstruation; Feminism; Medical Anthropology; Women's Health; Spain. 


\section{INTRODUCCIÓN}

La antropología médica o de la salud, centrada en el estudio de las mujeres y de los procesos de salud-enfermedad-atención que las afectan, sigue constituyendo una de las áreas principales del quehacer antropológico en el Estado español. Según Menéndez, los procesos de salud-enfermedad-atención son el "conjunto de representaciones y prácticas culturalmente estructuradas de las que cualquier sociedad se dota para responder a las crisis derivadas de la enfermedad y la muerte, donde también se incorporan los procesos de atención"(1).

Como ya advirtiera Mari Luz Esteban ${ }^{(2)}$ en su compilación de las principales tesis y trabajos realizados hasta el año 2010, uno de los temas más consolidados lo constituye la salud reproductiva. Si bien el panorama de la producción en antropología feminista de la salud debería ser completado, se presentan dificultades relacionadas con establecer los límites entre aquellos trabajos que abordan la salud y la gestión de los cuerpos de aquellos que son más próximos al parentesco, la sexualidad, el deporte, el cuerpo o las emociones. Otra dificultad descansa en que no todos los trabajos que se ocupan de las mujeres persiguen una intencionalidad feminista y, además, existen múltiples feminismos.

Desde nuestra perspectiva, si tuviéramos que delinear los principios de este subcampo de la antropología feminista de la salud, habría que partir de dos contribuciones decisivas: en primer término, la realizada por Inhorn ${ }^{(3)}$, hace ya una década, a partir de su revisión de más de 150 etnografías -solo en ingles- centradas en el estudio de la salud de las mujeres, en la que señala que las etnografías han realizado una serie de aportes que podrían ser agrupados en doce ejes:

- El poder de definir la salud de las mujeres.

- La esencialización reproductiva de las vidas de las mujeres.

- La construcción cultural de los cuerpos de las mujeres.
- El incremento de la medicalización de las vidas de las mujeres.

- El incremento de la hegemonía biomédica sobre la salud de las mujeres.

- La producción de la salud de las mujeres.

- Los efectos insalubres del patriarcado.

- La interseccionalidad en la salud de las mujeres.

- La intervención del Estado en la salud de las mujeres.

- Las políticas en la salud de las mujeres.

- La importancia de palabras morales locales de las mujeres.

- La importancia de comprender las subjetividades de las mujeres.

En segundo término, pero más relevante en nuestro contexto español, se hallan las contribuciones realizadas por Esteban, principalmente, en un artículo $^{(4)}$ publicado en Salud Colectiva, en el año 2006, en el que defiende la necesidad de un enfoque antropológico y feminista en el estudio de los procesos de salud-enfermedad-atención y, entre los postulados básicos, señala:

- la desnaturalización de las mujeres, de sus procesos corporales y su salud;

- la asunción de las limitaciones de la biomedicina;

- la incorporación de modelos complejos entre sexo, biología y cultura;

- el análisis del género (en relación con otros procesos de estratificación social) en los procesos corporales.

Ambas propuestas son coincidentes al señalar cuáles son los ejes de un análisis antropológico y feminista sobre los procesos de salud-enfermedad-atención, los cuales tomamos para abordar las vivencias y los significados de un grupo de mujeres acerca del ciclo menstrual en la Comunidad de Madrid. De esta manera proponemos que, aunque la salud reproductiva sea uno de los campos más desarrollados dentro de la antropología de la salud, aún quedan distintas realidades para analizar, sobre todo en la medida en que nos alejamos del embarazo y el parto, como experiencias que han eclipsado este campo ${ }^{(5)}$. 


\section{METODOLOGÍA}

El objetivo de esta investigación cualitativa ha sido una primera aproximación a las percepciones acerca del ciclo menstrual del grupo de mujeres, tratando de recabar sus visiones y experiencias cotidianas. El principal interés fue desvelar las subjetividades en torno a este proceso y cómo se relacionan con las condiciones sociales y culturales en las que se generan. Para ello se consideró que la técnica más adecuada era la entrevista abierta, en la que se pretende, a partir de un encuentro cara a cara entre la entrevistadora y la entrevistada, recoger las experiencias de las mujeres sobre su ciclo menstrual. Esta técnica permite un diálogo desde la visión subjetiva de cada mujer, dentro de una relación que permita expresar sus vivencias y opiniones en un marco de respeto a la intimidad y privacidad. Se realizaron 20 entrevistas con una duración de 60 a 90 minutos, a mujeres de 16 a 44 años que vivían en la Comunidad de Madrid. Se excluyeron aquellas con patologías graves, ginecológicas u otras, con tratamientos menstruales como métodos anticonceptivos, consumidoras de drogas, y deportistas de elite, pues estas circunstancias interaccionan en sus ciclos además de generar experiencias particulares que alejarían el estudio de una panorámica general. Las variables más relevantes de la muestra han sido: edad, nivel educativo, ocupación, tener o no hijos/as biológicos o haber usado o no métodos anticonceptivos hormonales.

La captación y el contacto fueron realizados por las propias investigadoras a través de redes informales. Se contactó telefónicamente a las mujeres y se les pidió su consentimiento, el cual se obtuvo sin dificultades, incluso muchas han manifestado su interés en participar pues les parecía muy importante y oportuno investigar sobre este tema, y consideraban estimulante compartir sus experiencias.

Se utilizó una grabadora digital para registrar los relatos, los cuales fueron trascritos literalmente para efectuar el análisis e interpretación del discurso. El análisis de la información se realizó a partir de las unidades de análisis establecidas. En la transcripción de los relatos se introdujeron nombres ficticios con el propósito de preservar el anonimato de los entrevistados.

\section{LOS MENSAJES DE UNA ANTROPOLOGÍA FEMINISTA DE LA SALUD RESPECTO AL ESTUDIO DE LA MENSTRUACIÓN}

Los mensajes que aquí presentamos no son exclusivos de la menstruación, sino que conforman las contribuciones o denuncias desde el feminismo antropológico de la salud que tristemente siguen presentes en las realidades estudiadas.

\section{Mensaje I. La gran paradoja: el ámbito reproductivo ha sido el más estudiado en la vida de las mujeres y sin embargo, existe una escasez de estudios sobre la menstruación}

El ciclo menstrual ha sido, sobre todo, objeto de estudio y análisis como proceso fisiológico que permite la reproducción biológica. Han sido especialidades sanitarias, como la ginecología y la matronería, las que mayormente han llevado a cabo estos trabajos. Sin embargo, su estudio se ha centrado en su inicio y fin: menarquia( ${ }^{(6)}$ y menopausia(7,8), obviándose cómo se produce a lo largo de este periodo, que ocupa casi la mitad de los ciclos vitales de las mujeres (de 35 a 40 años) $y$, por tanto, constituye un proceso cotidiano, habitual, ordinario. Otros acercamientos sanitarios se han centrado en lo que se ha definido como las patologías de la menstruación: los ovarios poliquísticos, la dismenorrea, el síndrome premenstrual(9) o la píldora anticonceptiva $^{(10,11)}$ con lo que nuevamente se prioriza el estudio de la anormalidad frente a la normalidad en el cuerpo de las mujeres.

Otras disciplinas sociales han ido más allá del interés por estudiar esta dimensión fisiológica y se han acercado a las representaciones sociales en torno al ciclo menstrual 
y las vivencias de las mujeres. Conocer estas perspectivas resulta relevante teniendo en cuenta la carga de prejuicios y tabúes que históricamente han estado ligados a la menstruación (y por ende al cuerpo de las mujeres). Algunos de estos estudios son: el trabajo pionero de Robert Snowden y Barbara Christian ${ }^{(12)}$ basado en un cuestionario acerca de patrones y percepciones sobre la menstruación en diez países diferentes (Egipto, India, Indonesia, Jamaica, México, Pakistán, Filipinas, Corea, Gran Bretaña y Yugoslavia); el de la politóloga Sophie Laws ${ }^{(13)}$, centrado en entrevistas a varones británicos; los estudios sobre experimentos psicológicos acerca de las percepciones de los productos higiénicos y de la misma menstruación ${ }^{(14)} ;$ y, por último, otros trabajos que se han acercado a las diferentes concepciones culturales de la menstruación así como sobre las explicaciones de los tabúes y regulaciones como los de Buckley ${ }^{(15)}$, Delaney ${ }^{(16)}$ y Knight ${ }^{(17)}$, que podrían ser clasificados como culturalistas, y nuevamente se alejan de la voz de las propias mujeres. Cabría aquí también incluir el texto clave de James Frazer ${ }^{(18)}$ La rama dorada en el que recoge distintos rituales, tabúes y representaciones de distintos grupos acerca de la sangre menstrual.

En el contexto del Estado español no han aparecido estudios que desde una perspectiva psicosocial indaguen en los saberes, experiencias y necesidades de las mujeres en torno a este proceso. De los pocos localizados destaca la tesis doctoral que está realizando Miren Guillo Arakistain ${ }^{(19,20)}$ bajo la dirección de Mari Luz Esteban Galarza. Sus objetivos se centran en analizar cómo ciertos colectivos de jóvenes vascas están resignificando la menstruación frente a la cultura médicocientífica hegemónica y a la representación negativa y/o naturalizada de la menstruación (incluido el tabú).

Se trata del primer estudio de estas características realizado en nuestro estado, el cual acompaña a otro estudio cuantitativo, más centrado en recoger las características del ciclo menstrual ${ }^{(21)}$. Esto muestra que, aunque en los últimos años hayan proliferado los textos y noticias en medios de comunicación acerca del ciclo menstrual, muchos de ellos con una fuerte vocación reinvindicativa y visibilizadora de esta realidad de la vida de las mujeres, no se han acompañado de un aumento de las investigaciones acerca del tema. Sin duda se habla más de la menstruación y se ha ampliado el discurso, pero daría la impresión de que se siguen repitiendo los mismos tópicos, se generalizan determinadas experiencias y visiones, se continúan omitiendo las voces de las propias mujeres y se olvida la necesidad de conocer en mayor profundidad y con mayor rigurosidad este proceso. Con el propósito de dar respuesta a este vacío del conocimiento surgió esta investigación.

\section{Mensaje II. La menstruación hace presente el cuerpo en la vida de las mujeres}

En las entrevistas, las mujeres señalan cómo la menstruación las hace conscientes de sus cuerpos. Esto lo van a expresar de distintas formas: unas señalando las imposiciones que pone el cuerpo menstruante en sus vidas; otras, mostrando las tensiones entre el cuerpo y sus deseos. En la primera situación están mujeres como Ana y Mercedes, a quienes la menstruación les obliga a hacer algunos cambios en sus vidas, escuchar y atender lo que les digan sus cuerpos, es uno de los aprendizajes que han ido haciendo a lo largo de sus vidas:

Hoy, por ejemplo, me vino antes de ayer la regla, quería haber ido al gimnasio ayer, no fui porque pensé: "Bueno es que justo me va a venir la regla, era una clase de estas de ciclo de bici, de... tal, y dije: "Creo que no puedo" porque era que no puedo, era como forzar un poco la maquinaria por así decirlo. (Ana, 37 años, estudios secundarios, trabaja como azafata, dos hijo/as)

Por ejemplo, lo que te contaba de hace un par de meses que tuve un pico de trabajo muy grande, se juntaría todo, quiero decir, porque no solo era esto, 
pero llevaba con una presión bastante alta en el trabajo dos meses, y como al mes y medio tuve que estar una semana fuera de casa en un hotel, además toda la semana en Barcelona trabajando y yendo a distintos sitios y después de trabajar en los centros por la tarde, claro, como no estaba en la oficina nunca, pues me tenía que poner a trabajar. Era una situación ya de por sí estresante, pero encima me vino la regla. Y es que me tiraba llorando todas las tardes una hora, o sea, digo, yo esto, aparte de por la presión mantenida y tal, digo, si no tengo la regla yo esto no me lo tomo tan mal, ¿sabes? Pero lo llevaba realmente mal, o sea, estaba... Y yo creo que también influyó, ¿sabes?, porque justo me vino. Entonces situaciones que normalmente yo puedo controlar, se me van un poquito más de las manos cuando estoy con la regla. (Mercedes, 32 años, estudios universitarios, trabaja en una empresa de ensayos clínicos)

Otras mujeres, como Elisa y Laura, reflexionan acerca de la influencia de las hormonas en sus cuerpos, en sus estados de ánimo; ellas tienen una postura crítica y abogan por negarse a atribuir de manera sistemática sus cambios emocionales a este hecho. El papel de las hormonas no sería algo estático e inamovible, sino que interacciona con agentes externos. El estrés, la sobrecarga laboral y/o por cuidados, etc., en definitiva, las condiciones de existencia y los eventos vitales influyen en el funcionamiento del sistema neuroendocrino. Desde esta posición aceptarían los cambios asociados al ciclo menstrual, pero negándose a asumir el determinismo biologicista que implica estar a merced de sus ciclos y de los cambios hormonales. Advierten que esta visión esencialista es un obstáculo para el desarrollo de un mayor control sobre sus cuerpos. Así lo explican:

Ahora estoy más cansada mentalmente antes de que me venga, unos días antes, pero ya no es por la regla y esto justifica todo, antes era un poco: estoy mal por la regla, y no intentaba trabajar ese sentimiento. Entonces ahora cuando me encuentro mal, digo: "puede ser por la regla, puede ser porque los niveles hormonales me estén cambiando, pero esto no significa que yo no tenga ningún poder sobre eso". Entonces, como que intento no justificarme con que estoy con la regla, porque también es eso de "déjame que estoy muy tonta porque estoy con la regla", es como si tú misma estás estigmatizando o no sé... dándole el juicio negativo a la regla. Entonces, bueno, hay días que estás triste o no, con o sin la regla, es independiente. A lo mejor te mueves más hormonalmente, que no es inamovible. Entonces eso sí, tengo más ansiedad los días que estoy con regla, hay veces que me duele $y$ entonces dejo de hacer cosas. Pero ya intento de no dejar de hacer cosas, que antes, al principio, sí. (Laura, 23 años, estudios universitarios, estudia formación profesional)

Creo, además, que no es algo que nos ocurra solo a nivel físico, sino que es algo que condiciona toda nuestra forma de estar también, pero que también podemos jugar con ello, todo lo que nos, nos..., a ver cómo lo digo... No es la causa de que seamos de cierta manera, sino que también tiene una síntomas que modifican nuestra forma de estar, pero que también podemos manejar, o sea yo no me puedo escudar en que "como soy mujer y tengo un ciclo menstrual, me pasa esto, me pasa lo otro". Claro que altera mi estado anímico y todos, pero yo también puedo controlar aquello, puedo jugar, soy dueña de mi cuerpo. (Elisa, 38 años, estudios universitarios, desempleada, tres hijos/as)

Los discursos de estas mujeres manifiestan la importancia de aceptar y pactar con las necesidades del propio cuerpo y con los cambios que pueden ir ligados al ciclo menstrual, superando así las visiones 
reduccionistas que han acompañado tradicionalmente a los estudios sobre los cuerpos de las mujeres.

\section{Mensaje III. La reafirmación de lo biológico como proceso cultural: las imbricaciones entre la biología y lo psicosocial en la menstruación}

Las mujeres afirman en distintos momentos de las entrevistas que, aunque la menstruación sea un proceso biológico, interactúa con otras circunstancias de sus vidas, sus condiciones, las visiones que han interiorizado sobre la menstruación, mostrando una relación dialéctica:

El estrés yo creo que no influye. Quizás... hombre, el estrés desde el punto de vista psicológico. A lo mejor el cansancio físico sí que puede influir. Es que no sé qué es antes, si el huevo o la gallina. Así que como me duele y me ha venido así como cruzada estoy mucho más agotada físicamente o que estoy muy agotada físicamente y lo llevo peor, ¿sabes?, no sé muy bien la relación, qué es lo primero. (Mercedes, 32 años, estudios universitarios, trabaja en una empresa de ensayos clínicos)

Aunque disponen de escasa información sobre los factores que influyen en el ciclo menstrual, afirman que habría distintas condiciones que agrupan bajo la categoría de estrés (como la sobrecarga, el exceso de trabajo, las épocas de exámenes, los problemas de relación...) que intervienen en estos cambios. Así, en los últimos años, están surgiendo estudios sobre la existencia de relaciones entre los factores ocupacionales, así como las condiciones medioambientales, con la salud, y especialmente con el ciclo menstrual, destacando la relación tan significativa con los xenoestrógenos. Sin embargo, estos discursos no se han difundido ni popularizado y las mujeres no los mencionan, ni siquiera aquellas que disponen de mayor información sobre el ciclo menstrual porque han estado en grupos o en colectivos activistas acerca de la menstruación. Nuevamente habría que plantear la escasez de investigaciones sobre estos factores y, específicamente, de aquellas que expliquen los mecanismos de estas interacciones ${ }^{(22)}$.

Volviendo a estos cambios que las mujeres perciben, pareciera que de alguna manera viven una especie de lucha y resistencia respecto a estos cambios, que remite a la idea de "cuerpo como campo de batalla", con toda la tensión y presión que esta puede generar. En este sentido, algunas mujeres proponen un cambio de actitud y cierta rebelión respecto a los modelos sociales que obligan a mantener un estado físico y emocional plano y lineal, un ánimo "positivo" y controlado de manera constante, una actividad y productividad sin tregua... Estos patrones propios de una sociedad capitalista, y profundamente enlazados con la masculinidad tradicional, acaban convirtiéndose en un impedimento para experimentar el dolor, la tristeza, la vulnerabilidad, el cansancio..., la amplia gama de emociones y posibilidades humanas:

Pues yo creo que habrá que desdramatizar. Quiero decir que es una cosa que, en general, me imagino que para la mayoría de las mujeres, es un estorbo. Quiero decir, que no es la situación ideal, pero como es algo con lo que vas a tener que estar viviendo cuarenta años de tu vida, aceptarlo como normal y aceptar que te puedas estar más triste unos días al mes, quiero decir, que no pasa nada, ¿sabes?, que ahora tendemos a querer estar siempre contentos, que todo sea fantástico, y no. Pues hay días buenos, hay días menos buenos y hay días regulares y uno tiene que aprender a vivir con eso. $Y$, en ese sentido, en el contexto de la menstruación, lo mismo. O sea, que sí. (Cristina, 28 años, estudios primarios, trabaja como esteticista)

Las mujeres incorporan en sus discursos la función de las hormonas en el ciclo menstrual, así como en las variaciones anímicas y físicas asociadas. Sin embargo, resulta llamativo que 
aluden a estas de manera vaga y genérica ya que, en realidad, cuentan con escasa información sobre el funcionamiento del sistema neuroendocrino y su relación con las distintas etapas del ciclo menstrual:

Yo supongo, digo, con tu ciclo menstrual hay... No sé porque hay muchas cosas que no sé cómo funcionan, pero hay un montón de hormonas y de todo, que se mueven y que hacen un poco pues que seas mujer. (Arantxa, 26 años, estudios universitarios, trabaja como fotógrafa)

O las hormonas, por ejemplo, te dicen: "Estás triste, eso es la regla". Pero te lo dicen, no te lo explican, ¿me entiendes? Tú te das cuenta, pero no te informan de eso, por ejemplo. (Mercedes, 32 años, estudios universitarios, trabaja en una empresa de ensayos clínicos)

Todas las mujeres recuerdan que en alguna de las clases de ciencias naturales les explicaron las fases del ciclo menstrual aunque ahora -en la entrevista- no sean capaces de repetir las características de cada una y las hormonas que intervenían. Todas reclaman que se tendría que dar una información más práctica y asequible. Si seguimos a la biología, se observa que el ciclo menstrual estaría conformado por dos conjuntos de procesos: por un lado, aquellos que tienen lugar en el ovario y el útero de la mujer -entre los que se encuentra la descamación y sangrado del endometrio del útero- $y$, por otro, los que se producen en distintos órganos del cuerpo. Ambos están regulados por la segregación de una serie de hormonas que interaccionan con determinados componentes neuroendocrinos (como neurotransmisores y otras hormonas) ocasionando que aparezcan cambios en distintos órganos y partes del cuerpo, lo que se conoce como la acción periférica. De ahí, que la médica endocrinóloga Carme Valls, hable de "la armonía del ciclo en el cuerpo de las mujeres" para indicar que las hormonas que habían sido asociadas tradicionalmente solo a la menstruación intervienen en numerosos procesos como los estados de ánimo, la termorregulación o la eliminación o acumulación de sustancias y líquidos. Sin embargo, en la mayoría de los manuales y libros solo se habla del primer proceso: aquel que tiene lugar en los ovarios y en el útero, reduciendo y simplificando la menstruación y generando así más confusión que certeza.

\section{Mensaje IV. Superando el esencialismo reproductivo: la menstruación no es solo un proceso reproductivo}

La menstruación ha sido conceptualizada, principalmente. como un proceso cuya utilidad y finalidad en el cuerpo de las mujeres es la reproducción, de ahí que también se conozca como ciclo reproductivo, y así figura en los principales manuales de ginecología, señalando su relación con la fecundidad. Afortunadamente, van apareciendo textos que mencionan que la menstruación tiene también otras funciones en el cuerpo de las mujeres, es un proceso "vital"(23). De este modo, se plantean dos posibilidades: los cuerpos de las mujeres funcionan bien con sus ciclos menstruales (como sugerimos anteriormente) y los ciclos menstruales no tienen como única y exclusiva función la fecundidad o maternidad, de manera que puede haber mujeres que tengan la menstruación y no quieran o no lleguen a ser madres. Sin embargo, algunas entrevistadas señalan que la única explicación que recibieron acerca de la menstruación es que esto les permitiría ser madres:

Y una de las cosas que me decía a mí mi familia, claro, es que lo asocio a eso. Me acuerdo que mi madre me decía: "Ya puedes tener hijos, ¿sabes?, puedes quedarte embarazada". Entonces lo asocio un poco a una imagen terrible que es ver a una niña con doce años, ¿no?, embarazada o teniendo relaciones sexuales, que me parece como completamente fuera de lugar. Entonces me parece como... me parece una expresión un poco fuera de lugar ¿sabes?, lo de mujer y tal. Pero 
por otro lado es verdad que te puedes quedar embarazada. Es decir, físicamente, estás más cerca de ser mujer que antes. (Estrella, 23 años, estudios universitarios, estudiante)

Sin embargo, la menstruación sigue presentándose como el proceso que permite ser madre aunque sea algo alejado en el momento de su llegada en la preadolescencia, y como si la fecundidad o la maternidad dependieran principalmente de este proceso biológico. Esta mirada de la menstruación, como un proceso reproductivo, también está anclada en todos los consejos que recibieron las mujeres acerca de que la menstruación marcaba el inicio de su reconocimiento como seres sexuales. Así se les indica que tienen que "cuidarse" o "guardarse", sin explicitar de qué ni cómo, aunque en ocasiones se señala: "de la sexualidad de los hombres", conformando una experiencia de temor acerca de los hombres, de sus cuerpos y de las relaciones sexuales:

$Y$ encima te cuentan todo eso y te lo cuentan de esa manera de "ya te tienes que guardar" ¿no?, "ya tienes que tener mucho cuidado" isabes? Pues eso, pues es que es lo de antes ¿sabes? Y mi madre no era mayor, pero sí que en ese sentido era muy clásica. Pues eso, porque se lo habían inculcado así a ella. (Lola, 41 años, estudios secundarios, ama de casa, dos hijo/as)

Nada, que ya había que tener cuidado con las relaciones, y con todo, porque ya era diferente. (Mercedes, 32 años, estudios universitarios, trabaja en una empresa de ensayos clínicos)

Si bien es cierto que la menstruación viene a marcar que el cuerpo de las mujeres se ha desarrollado y que sus genitales están maduros no solo para la reproducción sino también para la sexualidad, esto debería conducir a interrogarnos acerca de que a estas edades (incluso previamente) habría que empezar a hablar de sexualidad con las niñas y, habría otro desafío sobre cómo proponer discursos que reconozcan que una de las funciones podría ser la posibilidad de quedarse embarazada pero desde marcos más positivos, que no asociaran el cuerpo con algo a ocultar, o la sexualidad como algo negativo que solo va a traer peligros (como el embarazo). Se proyecta entonces sobre las niñas y adolescentes el miedo a un embarazo no deseado, pero sin construir con ellas posibilidades y recursos para protegerse e ir construyendo sus propias opciones y decisiones. Este tipo de comunicación está repleta de alusiones veladas y opacas a la sexualidad, pero sin hacer referencias claras y explícitas, y sin establecer un verdadero diálogo.

Las mujeres entrevistadas ante este nudo quieren disponer de explicaciones e información, procedentes tanto del ámbito sanitario como educativo, así como de la sociedad en general, que les permita hablar y ver sus menstruaciones como procesos normales. Ellas saben, aunque no puedan explicar cómo, que sus cuerpos están bien cuando les viene la menstruación:

Si te viene la regla es que tu cuerpo está bien. O sea, si no te viene la regla... Hombre, puede ser que estés embarazada ¿no? Pero si no estás embarazada y no te viene la regla, algo pasa. (Estrella, 23 años, estudios universitarios, estudiante)

\section{Mensaje $V$. El inicio de la medicalización de las vidas de las mujeres a partir de la menarquia}

Aunque se ha escrito mucho sobre el proceso de medicalización de las vidas de las mujeres, esta aproximación a la menstruación pone de manifiesto cómo las intervenciones educativas, sociales, culturales y sanitarias se han dirigido tanto a controlar los cuerpos de las niñas-mujeres como a incorporarlas bajo el conocimiento experto. Los mensajes que recibieron las niñas-mujeres cuando les llegó la menstruación se han centrado en que a 
partir de ese momento tienen que dejar de ser niñas y asumir ciertas responsabilidades que conlleva el ser mujeres, muchas de las cuales se centran en la higiene:

Demasiado rápido para mí. Fue como que yo me seguía sintiendo una niña, tenía que afrontar que ya no era una niña y tenía que hacer cosas de mujer, digamos: la depilación, comprar compresas, tomarme los Ibuprofeno para que no me duela, porque mi menstruación a veces es muy dolorosa, de vez en cuando. (Almudena, 18 años, estudios secundarios, estudiante)

Si sabía lo que significaba y tenía que tener cuidado: una buena higiene, un buen control para que aquello no desbordase. (Elisa, 38 años, estudios universitarios, desempleada, tres hijos/as)

Entre las responsabilidades que conlleva el paso a ser mujer se encuentran que sus menstruaciones no solo no se noten, sino que sean invisibles e inoloras, de ahí todas las prescripciones higiénicas (enfatizadas y recreadas en la publicidad existente en los medios de comunicación). Aquí aparece cómo se van incorporando estas cargas con el tabú cultural e histórico que asocia menstruación con suciedad e impureza. El tabú también se manifiesta en que los cuidados higiénicos y del dolor o las molestias que causa la menstruación son los temas mayormente legitimados a la hora de construir relatos sobre la menstruación. La insistencia en la higiene realza la visión de la mujer limpia que trata de eliminar las impurezas de su vida. En relación con las ideologías cristianas, el dolor y las molestias se articulan con la idea de que el cuerpo de las mujeres está diseñado para el sufrimiento, para ser vivido más desde la enfermedad y así precozmente las niñas se convierten en dolientes y consumidoras de fármacos que alivien estas molestias (como decía una de las entrevistadas, resulta común que les ofrezcan a la vez la compresa o el tampón y el ibuprofeno). Entonces cabría cuestionarse ¿dónde está la normalidad y la salud del ciclo menstrual? ¿No sigue siendo la menstruación uno de los bastiones de la medicalización de los cuerpos de las mujeres?

Otra forma en que se inicia esta medicalización, según nos refieren las mujeres entrevistadas, es que a partir de la menarquia, sus madres las animan a empezar las visitas al sistema sanitario, como una más de las responsabilidades que tienen que ir incorporando en sus vidas. Este hecho muestra cómo las mujeres tempranamente empiezan a desarrollar una cultura de autocuidado que conlleva no solo a iniciar un seguimiento preventivo ginecológico sino también iniciar una observación y registro de las características de sus menstruaciones, principalmente, a través de aplicaciones informáticas. Con esta información las mujeres acuden al personal sanitario experto para que les informe si todo lo que sucede en su cuerpo funciona correctamente. Sin embargo, en el sistema sanitario las respuestas que encuentran son bien distintas. Allí la única respuesta que reciben es que "todo es normal", lo cual puede retrasar el diagnóstico de problemas de salud (carencias nutricionales, exposición a disruptores endocrinos) y de enfermedades, como puede ser la endometriosis. Probablemente, esto también esté en relación con la falta de información del personal sanitario sobre las características del ciclo menstrual saludable. De modo que, ante cualquier demanda o problema de la mujer como dolor, sangrado abundante e irregularidades, la única respuesta es la prescripción de la píldora anticonceptiva, iniciándose su consumo a edades tempranas y manteniéndose durante muchos años, siendo uno de los más claros ejemplos de medicalización de la vida de las mujeres.

\section{Mensaje VI. Hacia la superación de visiones patológicas del cuerpo de las mujeres}

Emily Martin ${ }^{(24)}$ es una de las primeras antropólogas en plantear, desde un brillante examen marxista, cómo los cuerpos de las mujeres, particularmente como cuerpos reproductivos, han sido disciplinados en el siglo XX. Las nociones de producción y 
productividad penetraron en los textos médicos y en las prácticas sanitarias creando una visión negativa de los cuerpos de las mujeres. En nuestra investigación, al preguntar a las mujeres acerca de los imaginarios y significados del ciclo menstrual, encontramos que sus respuestas se asemejan a un caleidoscopio, es decir, integran distintas visiones que recogen las múltiples facetas que conlleva la menstruación en sus vidas, así como las encrucijadas y contradicciones. Por un lado, habría una serie de visiones que señalan -y reivindican- una visión de normalidad y naturalidad acerca de la menstruación, presentes en todas las mujeres aunque algunas más como propuesta ideal que como una experiencia real. Próxima a estas visiones están las que relacionan menstruación con salud y con juventud. Por otro lado, en otras prima la representación de ser una carga, una incomodidad, algo desagradable y vergonzoso. La vergüenza muestra que la menstruación sigue siendo un tabú, y no es entendida como un proceso fisiológico propio del cuerpo de las mujeres sino como algo sucio y desagradable que debe ser escondido y convierte a la higiene y sus cuidados en un imperativo que sobrecarga a las mujeres. Pero no solo es debido a que la sangre sea algo sucio, es más complejo:

Claro, es como... Es dos partes, es decir, es una sangre y las sangres son malas, son asquerosas y son sucias y además encima porque viene de la vagina. Entonces claro, la vagina es algo que hay que lavar, que huele mal, que es íntimo, etcétera. Entonces si viene de ahí, es feo eso, es horrible ¿no? Entonces bueno, entre que la sangre en general es sucia y que además proviene de los genitales, es la peor sangre, es decir, estoy segura, eso, que cualquier persona es capaz antes de chuparte una herida de la mano porque tal antes de poder chupar o tocar... La sangre, por ejemplo, de los brazos se toca. Los médicos, etcétera, tal, o incluso la gente "¿estás sangrando?". Pero nadie te toca la sangre de la regla porque es algo asqueroso, cerdo. Incluso, yo con la copa de luna, volví a descubrir muchas... muchísimas reacciones ¿no?, de cómo se vive ahora. Porque claro, la copa de luna no es fácil meterla, tienes que manipular mucho los genitales, ponértela, al principio te tienes que acostumbrar un poquito a tenerla ahí, y al sacar... Y claro, mis amigas, sobre todo las de mi pueblo, que son las de toda la vida y eso, "iqué asco, cuando sacas eso!", y digo, ves cómo siempre las reacciones "ipero qué asco, porque luego ahí toda la sangre!", y lo han rechazado y siguen poniéndose otro tipo de... pues las compresas y los tampones. (Uxía, 30 años, estudios universitarios, trabaja como psicóloga)

Lógicamente, estos prejuicios atávicos (suciedad, vergüenza, repulsión...) potencian la relación del malestar asociado al ciclo menstrual. Uxía y Claudia reflexionan con enorme lucidez sobre el impacto de los mensajes negativos y la desinformación en las experiencias de las mujeres:

Siempre tuve muy claro que yo lo he vivido de forma triste, pero es que también no había otra opción de vivirlo, porque yo, todos los mensaje que me han transmitido y todo lo que ha rodeado a la menstruación era negativo. Entonces yo, nadie, ninguna amiga mía, ni ninguna persona me habló de la menstruación de forma positiva, entonces, claro, ¿Cómo iba a reaccionar? (Uxía, 30 años, estudios universitarios, trabaja como psicóloga)

A mí me parece que el enfoque que tengas hacia cualquier cosa de tu cuerpo, pues por ejemplo darle esa naturalidad $y$ esa normalidad, hace que se viva de diferente manera. A mí me parece que también hay ahí un..., no sé, una connotación a lo mejor de cómo lo vives, ¿no? Cómo se ha introducido en tu vida, cómo llegó a ti, cómo las siguientes experiencias que tienes cómo las vives. O sea, me parece que eso es fundamental, con 
lo cual, a lo mejor si hubiera otro entendimiento de qué es realmente la menstruación, para qué sirve, cómo lo puedo manejar o vivir, pues a lo mejor esas personas no la vivirían de esa manera tan traumática. (Claudia, 41 años, trabaja como peluquera, dos hijo/as)

Ninguna de nuestras entrevistadas formaba parte del movimiento activista menstrual contemporáneo ${ }^{(25)}$. Sin embargo, tres mujeres se habían acercado a estos grupos que tienen una misión común: borrar la vergüenza menstrual y el estigma a través de la creación de una revolución roja basada en el ecologismo, la feminidad y la salud, cuyo inicio podríamos situarlo en la traducción y publicación del libro Luna Roja de Miranda Gray en nuestro país. Sin embargo, hay que señalar que las mujeres entrevistadas cercanas al feminismo son las que expresan una mayor conciencia, reconocimiento y espíritu crítico frente a estos distintos tabúes que impelen a vivir conflictivamente su condición de mujeres y son las que están haciendo procesos de resignificación y desplazamiento de los mandatos, normas y prejuicios más habituales. Pero además, ninguna de ellas defiende una visión esencialista de la menstruación.

\section{Mensaje VII. La diversidad entre las mujeres}

Otro de los elementos que surgen de esta limitada investigación estriba en la diversidad hallada entre las mujeres respecto al fenómeno estudiado, la cual se refleja en múltiples hechos. Por un lado, en sus relatos sobre la menarquia que van desde la aceptación con naturalidad de un hecho esperado, pasando por la confusión y la extrañeza por el cambio producido, hasta reacciones de rechazo; sus respuestas ante este hecho están influenciadas por la edad que tenían, por la preparación previa, por las respuestas de las personas más próximas a ellas en ese momento y por el entorno sociocultural. Es interesante comprobar cómo la construcción de significados en torno a la menarquia se produce en un contexto de intersubjetividad, en el que a través de las interacciones con las personas significativas se van construyendo las representaciones sobre la menstruación.

Por otro lado, la pluralidad de sentidos con los que vinculan la menstruación y la femineidad. La mayoría critica enérgicamente esta identificación simplista de la menstruación con el hecho de ser mujer. Algunas rechazan que este proceso biológico sea el que mayormente defina su identidad y marque la diferencia sexual:

\section{Vamos, yo creo que es algo antiguo, que siempre se ha dicho: "ya eres mujer". Pero como digo yo, qué pasa, que antes eras una hermafrodita. (Candela, 43 años, estudios primarios, trabaja como limpiadora, dos hijos/as)}

También porque, en el camino de conversión de niña a mujer adulta, la menarquia es un ingrediente más del desarrollo, las entrevistadas consideran que ser mujer es algo más complejo, que se conforma a través de distintas experiencias en las que la menstruación no sería el hecho principal:

\begin{abstract}
Ahora tengo una amiga que está haciendo una obra de teatro sobre la mujer y se hicieron la pregunta de ¿en qué momento te sentiste por primera vez mujer? Y me contó las experiencias de ese grupo y ninguna lo asociaba a la regla. (Laura, 23 años, estudios universitarios, estudia formación profesional)
\end{abstract}

Sin embargo, saben que hay algo sobre lo que debemos seguir pensando y es que la menstruación, los mandatos, los estereotipos y las vivencias que se derivan de ello, es algo que específicamente les sucede a las mujeres, no a todas (hay mujeres que no tienen la menstruación y hay mujeres transgénero que tampoco) y no siempre, solo durante una parte de sus vidas (de la menarquia a la menopausia y con etapas en las que pueden no tener menstruación), aunque su huella perviva más tiempo. $Y$ esto que atañe de forma directa a muchas mujeres, las 
puede colocar en un lugar de vulnerabilidad y dependencia, desde el cual es más fácil su subordinación y control en aras del mantenimiento de un sistema que quiere que, entre otras cuestiones, sigan siendo reproductoras y asexuales.

Quizá una antropología feminista de la salud, en la actualidad, debería mantener una actitud crítica que promueva la alteración de las desiguales relaciones de género. Una forma de iniciar este camino fue revisar algunos de los campos consolidados como la salud reproductiva, y a partir de las contribuciones realizadas por Inhorn ${ }^{(3)}$ y Estebann ${ }^{(4)}$, analizar los datos procedentes de esta investigación cualitativa sobre el ciclo menstrual. De este modo, encontramos cómo el ámbito reproductivo, a pesar de ser el más estudiado en la vida de las mujeres, presenta numerosas carencias en el acercamiento a procesos como la menstruación y, particularmente, desde ciertas perspectivas centradas en las experiencias cotidianas de las mujeres. Además, en la vida de las mujeres, la menstruación hace presente sus cuerpos, los que están construidos en una interacción constante y compleja entre la biología, la cultura y lo social, entre lo que les acontece, lo que quieren y sus condiciones de vida. Las mujeres entrevistadas tratan de superar la esencialización reproductiva así como el incremento de la medicalización en sus vidas, que puede iniciarse a partir de la llegada de la menstruación. Sin embargo, no es una cuestión trivial, cuando han sido socializadas en visiones patológicas sobre sus cuerpos y cuando la biomedicina hegemónica, al igual que el patriarcado, tratan de mantener una visión monolítica sobre las mujeres, que hemos de ir rompiendo con ejercicios como el de este artículo.

\section{AGRADECIMIENTOS}

El estudio fue financiado a través de una beca de la Cátedra de Investigación Antonio Chamorro y Alejandro Otero, Facultad de Medicina, Universidad de Granada.

\section{REFERENCIAS BIBLIOGRÁFICAS}

1. Menéndez E. El modelo médico y la salud de los trabajadores. En: Basaglia F. La salud de los trabajadores. México: Nueva Imagen; 1978. p. 11-53.

2. Esteban ML. La investigación antropológica en salud, género y atención en el Estado Español: tesis doctorales y grupos de investigación. En: Esteban ML, Comelles JM, Diez Mintegui C. Antropología, género, salud y atención. Barcelona: Bellatera; 2010. p. 329-345.

3. Inhorn M. Defining women's health: A dozen messages from more than 150 ethnografies. Medical Anthropology Quartely. 2006;20(3):345-378.

4. Esteban ML. Las ventajas de un enfoque antropológico y feminista. Salud Colectiva. 2006;2(1): 9-20.
5. Blázquez-Rodríguez M. Aproximación a la Antropología de la Reproducción. Revista AIBR 2005;42:1-25.

6. Fernández Olguín D. Los tabúes de la menarquia: un acercamiento a la vivencia de jóvenes escolares chilenas. Revista de Psicología. 2012;21(1):7-29.

7. Francés Ribera L. Análisis sobre los modelos culturales de la menopausia. [Tesis de doctorado]. Barcelona: Departamento de Antropología Cultural e Historia de América y África. Facultad de Geografía e Historia, Universitat de Barcelona; 2003.

8. Puialto-Durán MJ, Moure-Fernández L, SalgadoÁlvarez C, Antolín-Rodríguez R. Conocimientos sobre la menopausia en las mujeres de 30 a 60 años del área sanitaria de Vigo. Atención Primaria. 2011;43:269-270.

9. García Porta M. Síndrome premenstrual (SPM): Aproximación crítica. AIBR: Revista de Antropología Iberoamericana. 2006;1(1):80-102.

10. Sánchez-Borrego R, García-Calvo C. Spanish women's attitudes towards menstruation and use of a continuous, daily use hormonal combined contraceptive regimen. Contraception. 2008;77(2):114-117. 
11. Shipman Gunson J. "More natural but less normal": Reconsidering medicalisation and agency through women's accounts of menstrual suppression. Social Science \& Medicine. 2010;71:13241331.

12. Snowden R, Christian B. Patterns and perceptions of menstruation: A World Health Organization international collaborative study. London: St. Martin's Press, World Health Organization; 1983.

13. Laws S. Issues of blood: The politics of menstruation. Hampshire: Macmillan; 1990.

14. Patterson AS. The menstrual body. New Orleans: University of New Orleans; 2013.

15. Buckley T, Gottlieb A. Blood magic: The anthropology of menstruation. Berkeley: University of California Press; 1988.

16. Delaney J, Lupton MJ, Toth E. The curse: a cultural history of menstruation. Illinois: University of Illinois Press; 1988.

17. Knight C. Blood relations: menstruation and the origins of culture. New Haven: Yale University Press; 1991.

18. Frazer JG. La rama dorada [1890]. Barcelona: Fondo de Cultura Económica; 1981.
19. Guillo Arakistain M. La incorporación de la investigación: políticas de la menstruación y cuerpos (re)productivos. Nómadas. 2013;39:233-245.

20. Guillo Arakistain M. Mujeres jóvenes y menstruación: contracultura y resignificación del ciclo menstrual en el País Vasco. En: Romaní O, Casadó L, (eds.). Jóvenes, desigualdades y salud: Vulnerabilidad y políticas públicas. Tarragona: Publicacions URV; 2014. p. 143-165.

21. Barranco E, Jiménez-Díaz LM, IribarneDurána $\mathrm{O}$, Ocónc E, Salamanca MF, Fernández $\mathrm{N}$, Olea $\mathrm{E}$. Determination of personal care products -benzophenones and parabens- in human menstrual blood I. Journal of Chromatography B. 2016;1035:57-66.

22. Valls Llobet C. Mujeres invisibles. Barcelona: DeBolsillo; 2006.

23. Nelson LM, Hillard P, Trott J, Khosla S, Warren MP. The menstrual cycle-getting the "vital" respect it deserves [Internet]. 2003 [citado 10 oct 2016]. Disponible en: http://tinyurl.com/ y7qeamgx.

24. Martin E. The woman in the body: A cultural analysis of reproduction. Boston: Beacon; 1987.

25. Bobel C. New blood: Third wave feminism and the politics of menstruation. New Jersey: Rutgers University Press; 2010.

FORMA DE CITAR

Blázquez Rodríguez M, Bolaños Gallardo E. Aportes a una antropología feminista de la salud: el estudio del ciclo menstrual. Salud Colectiva. 2017;13(2):253-265. doi: 10.18294/sc.2017.1204.

Recibido: 31 de octubre de 2016 | Aprobado: 17 de febrero de 2017

Este obra está bajo una licencia de Creative Commons Reconocimiento-No Comercial 4.0

Internacional. Reconocimiento - Permite copiar, distribuir y comunicar públicamente la obra.

A cambio, se debe reconocer y citar al autor original. No Comercial - Esta obra no puede ser

utilizada con finalidades comerciales, a menos que se obtenga el permiso.

http://dx.doi.org/10.18294/sc.2017.1204 